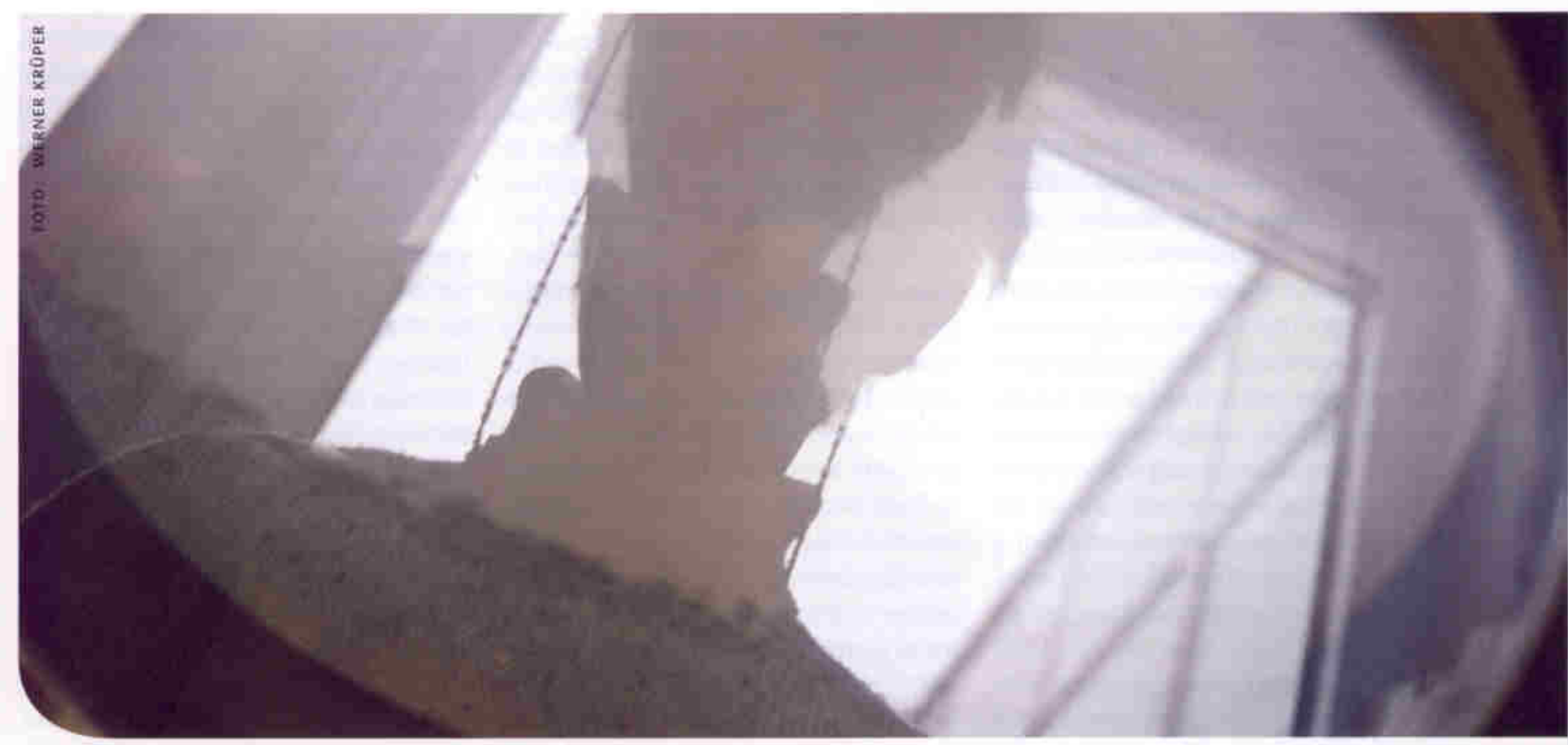

\title{
„Mein Mann hatte Demenz“ - von den Erfahrungen und der Sichtweise einer Angehörigen
}

\section{Daniela Händler-Schuster, Michael Schulz}

Die Demenzerkrankung stellt das Gesundheitswesen in der Schweiz vor eine große Herausforderung. In der Schweiz erkranken gemäß den Ausführungen der Schweizerischen Alzheimervereinigung (2009) jährlich rund 24.700 Menschen neu an einer Demenz, wobei das Risiko mit zunehmendem Alter steigt. Allein bei den über 90 -jährigen Menschen ist pro Jahr mit 4.000 Neuerkrankungen zu rechnen. Laut Medienmitteilung des Bundesamtes für Statistik (2009) ist die Demenzerkrankung im Jahr 2007 erstmals als die dritthäufigste Todesursache beschrieben worden, wobei die eigentliche Todesursache in den Folgen der Demenzerkrankung liegt.

\section{Hintergrund}

Die Demenz vom Alzheimer-Typ wird nach der Internationalen Klassifikation für Krankheiten (2010) definiert als eine primär degenerative zerebrale Krankheit mit unbekannter Átiologie und charakteristischen neuropathologischen und neurochemischen Merkmalen. Sie beginnt meist schleichend, entwickelt sich langsam, aber stetig über einen Zeitraum von mehreren Jahren und wird als eine komplexe neuropsychiatrische Störung bezeichnet, die sich durch einen zunehmenden Gedächtnisverlust manifestiert. Schwierigkeiten lassen sich im Rechnen, in der Lernfähigkeit, im Sprechen und in der Urteilsfähigkeit finden. Vor allem das Kurzzeitgedächtnis und das Denkvermōgen sind eingeschränkt. Nach den Ausführungen von Dibelius und Uzarewicz (2006) geht die Erkrankung mit einem Verlust erworbener Fähigkeiten, z.B. in der Handlungs- und Planungskompetenz. einher. Beeinträchtigungen im Sehen, im Hören und im Tasten machen es demenzerkrankten Menschen schwer, sich adăquat auf ihre Umgebung einzustellen. Gefahren im Alltag können nicht mehr richtig eingeschätzt werden. Mitunter ist auch die Persönlichkeitsstruktur betroffen, was besonders für Angehörige schwer ist, z.B. dann, wenn sie nicht wiedererkannt werden.

Ein wesentlicher Teil der pflegerischen Betreuung Demenzerkrankter wird in der Schweiz von den Angehörigen geleistet. Dabei liegt nach Schätzungen von Höpf- 
linger (2004) der Anteil der pflegenden Angehörigen, die zum Teil mit professioneller Unterstützung zu Hause pflegen, bei $55 \%$, maximal bei $60 \%$. Perrar et al. (2009) berichten in einer australischen Studie, dass der größte Anteil an Pflegeleistungen von Angehörigen und nicht von professionell Pflegenden erbracht wird. Öber die Hälfte (60\%) aller Menschen mit Demenz und immerhin noch $30 \%$ aller Menschen im fortgeschrittenen Stadium der Erkrankung werden durch Angehörige zu Hause betreut. Dass immer hăufiger ältere Menschen ihre Partner betreuen und diese Angehörigen vor eine große Herausforderung gestellt sind, bekräftigen auch die Zusammenfassungen von Kofahl, Lüdecke und Döhner (2009).

Wenn Pflegende Demenzerkrankte betreuen, dann haben sie es nicht nur mit den einzelnen Menschen, sondern in der Regel mit Systemen zu tun. Ehepartner, die Familie im Allgemeinen, aber auch Freunde bleiben von einer demenziellen Entwicklung nicht unberührt, auch sie müssen einen Weg finden, die Erkrankung des anderen zu verstehen. Nach Corbin und Strauss müssen Menschen mit chronischen Krankheiten unterschiedliche Formen von Arbeit leisten, damit eine Anpassung an die sich stark ändernden Lebensumstände gelingen kann (1993). Die Autoren sehen die Schwerpunkte der Arbeit von chronisch kranken Menschen und ihren Familien vor allem in der krankheitsbezogenen Arbeit, in der biografischen Arbeit und in der Alltagsarbeit. Wegen der Multidimensionalität der Faktoren, die das Krankheitsgeschehen beeinflussen, gehen Corbin und Strauss in ihrem Krankheitsverlaufskurven-Modell davon aus, dass die Versorgung von chronisch kranken Menschen immer wieder angepasst und gemanagt werden muss. Hierbei hat der Einbezug von Familienangehörigen sowie von allen an der Versorgung Beteiligten eine hohe Bedeutung. weil diese auf den Krankheitsprozess einen Einfluss nehmen (Corbin und Strauss 1993. S. 29f; Schulz, Dorgerloh, Ratzka, Gray und Behrens 2007). Pflegende Angehörige verfügen über eine besondere Form des Expertenwissens. Sie haben in vielen Fällen kunstvolle und wirksame Strategien entwickelt, damit ein weiteres Zusammenleben möglich ist, was sich häufig positiv auf die Lebensqualität der Demenzerkrankten auswirkt. Angehörige sind diejenigen, die in der Regel am besten Bescheid wissen über die Gewohnheiten der Erkrankten. Sie können uns Pflegenden insbesondere bei Menschen mit kognitiven Beeintrăchtigungen eine wertvolle Stütze sein, unter anderem bei der Erhebung der Anamnese. Für professionelle Pflegende sind die Erfahrungen von pflegenden Angehörigen nicht nur wichtig, um den Gesamtzusammenhang von Erkrankungen zu verstehen, sie können die Informationen nutzen, um Maßnahmen im Umgang mit den Demenzkranken gezielt zu planen, durchzuführen und entsprechend dem Pflegeprozess zu evaluieren.

Die Gründe für Familienmitglieder, ihre Angehörigen mit Demenzerkrankung zu Hause zu pflegen, können unterschiedlich sein. „Miteinander verheiratet sein" und weil man „füreinander da sein möchte" sind laut einer Untersuchung von

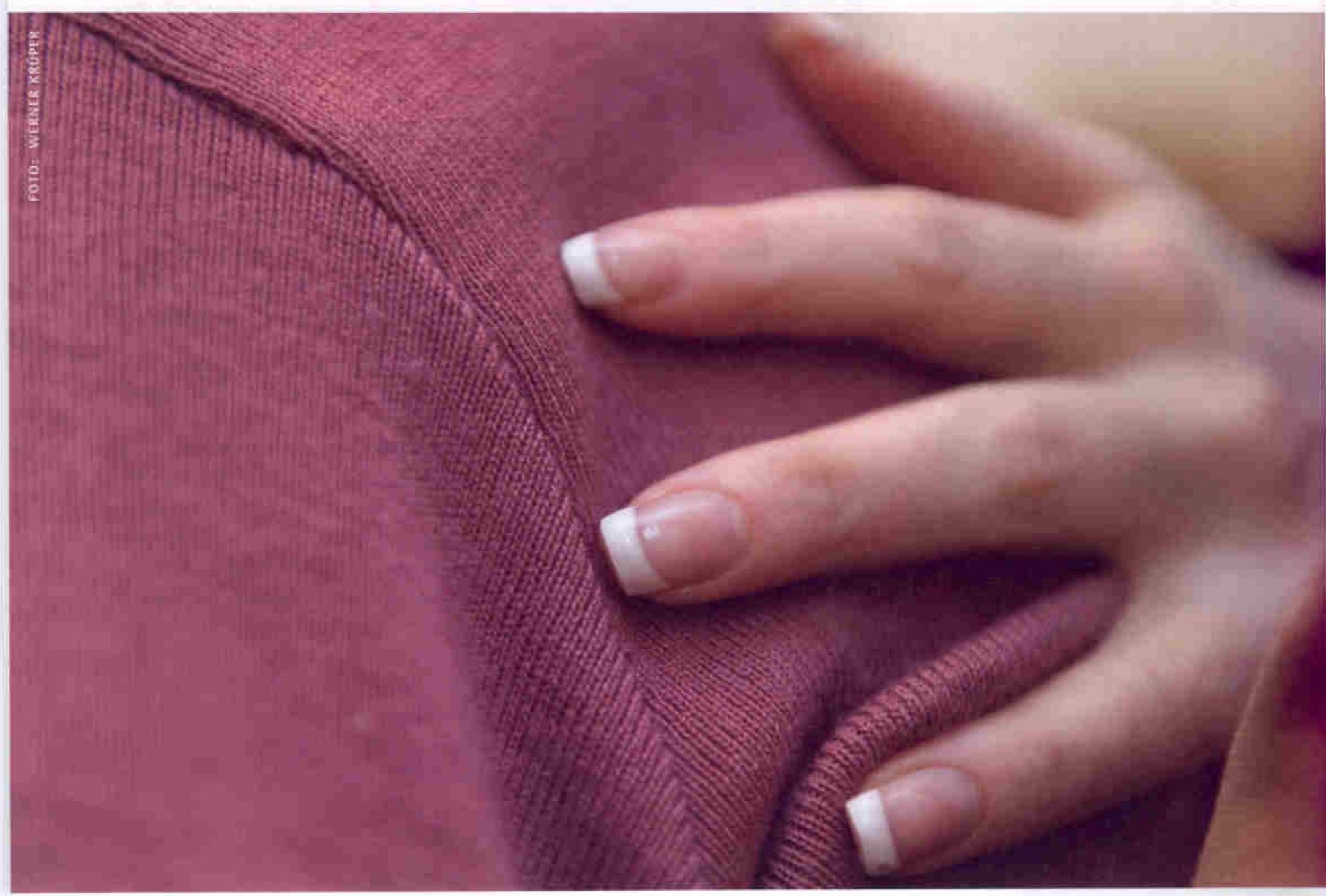


Oppikofer (2009) zwei der Hauptbeweggründe. Pflegende Angehörige können in ihrer Pflegerolle ein gewisses Maß an Zufriedenheit empfinden, was auch einen gewissen Stolz bei ihnen auslösen kann. Die Präsenz, die eine Pflege von Menschen mit Demenz pflegenden Angehörigen abverlangt, kann allerdings auch sehr einschrânken und die Angehörigen von ihrer Umwelt isolieren.

Nach Oppikofer (2009) können Gefühle der Entfremdung gegenubber dem Demenzerkrankten auftreten, sowie eine emotionale Distanz. Durch die intensive Zeit, welche die Angehörigen aufbringen, kann es dazu kommen, dass sie inner- und auBerfamiliäre Kontakte vernachlässigen oder aufgeben. Es kann dazu kommen, dass pflegende Angehörige eine geringe Unterstützung durch die Familie selbst erfahren. Die negativen Konsequenzen, die mit den vielen Belastungen einhergehen, beeintrăchtigen das psychische Wohlbefinden und können zu Ärger, Frustration, Schuldgefühlen und Unzufriedenheit führen, gleichzeitig können sie Schlafstörungen und Erschōpfungszustände auslösen. Uns [den Autoren] stellt sich die Frage, wie pflegende Angehörige rückblickend ihre Situation beschreiben.

\section{Von der Vernissage zum Interview}

Nachdem wir von der Vernissage von Frau L. zum Thema „Alzheimer - Sichtweise einer Partnerin" erfahren hatten, fragten wir uns, wie Frau L den Krankheitsverlauf ihres Mannes erlebt hatte.

Frau L pflegte ihren Mann, der an Alzheimer erkrankt war, 16 Jahre bis zu seinem Tod im Jahr 2006. In der Zeit der Alzheimer-Erkrankung war Herr L. anderthalb Jahre in einem Altenheim, ansonsten lebte er mit seiner Frau in der gemeinsamen Wohnung in Zürich.

Herr L war bis zu seiner Pensionierung in einer Firma der Metallbranche tätig, in leitender Position. Es war eine sehr umfangreiche Tätigkeit, die ihn immer auch wieder ins Ausland reisen ließ. Er kaufte Rohfabrikate ein, die er auch wieder in Schweizer Firmenbesitz verkaufte. Er organisierte Transporte mit Lastwagen und hatte mit verschiedensten Menschen zu tun, was ihm sehr gelegen hatte. Herr L. war verwitwet, als er Frau L. kennenlernte. Erst nach einigen Jahren der Zweisamkeit entschlossen sich beide, ein erneutes Mal zu heiraten und eine gemeinsame Wohnung zu beziehen. Beide hatten bereits zwei erwachsene Kinder, die ihre eigenen Wege gingen.

Herr und Frau L erfüllten sich im Jahre 1991 den Wunsch, auf einen anderen Kontinent zu reisen. Im Jahr 1992 waren sie dann das letzte Mal am Meer, bevor sie die Diagnose erfuhren, dass Herr $L$ an Demenz erkrankt war. Das Wissen um die Diagnose stimmte Herrn L oft depressiv. Frau L. erinnert sich, dass sie das Glück hatten. im Herbst noch auf ein Kreuzfahrtschiff zu gehen und so der Tatsache der Krankheit auszuweichen, was ihnen beiden sehr gut tat. Im Mittelmeer wärmte sie die Sonne, und das Meer, das Herr L. so liebte, half ihnen, mit der Diagnose „Demenz" zu leben. Beide wanderten gerne und viel, auch noch mit der Diagnose im Bewusstsein wanderten sie täglich. Im Jahre 1993 wurde Frau L. frühzeitig pensioniert. Sie war in einer Bank angestellt und überlegte sich bei ihrer Pensionierung, wie es weitergehen solle mit ihr und ihrem Mann, der zusehends Einbußen durch die Erkrankung hatte und vermehrt Unterstützung im Alltag benötigte. Sie fragte sich, wie lange das wohl noch gehen würde.

Die wenigen Informationen aus diesem Einzelfall geben Gelegenheit zur Betrachtung. Eine Frau pflegt ihren Mann und überlebt ihn. Herr L. hat den größten Teil der zu vollbringenden Pflege im häuslichen Umfeld von seiner Ehefrau erfahren. Wir fragten Frau L. vor ihrer Vernissage in einem Brief, ob sie bereit sei, mit uns über ihre Erfahrungen zu sprechen. Wir dachten, dass eine Person mit solchen Erfahrungen auch Pflegenden mit Schwerpunkt Psychiatrie einiges zu erzählen habe.

Wir erhielten von Frau L. einen Brief, in dem sie von der Krankheit ihres Mannes berichtete. Sie schrieb von Zeiten der Wut, der Aggression und der Verzweiflung, welche sie nicht negieren konnte, aber auch immer wieder von den schönen Momenten, die mit der Erkrankung verbunden waren. Sie schrieb von den Gewohnheiten ihres Mannes, an denen sie sich zu orien- tieren versuchte. Da war beispielsweise das Pfeifen ihres Mannes, das über seine Stimmung Auskunft gab und sie orientierte, wo er sich gerade befand. Was ihr besonders geholfen hatte, waren die Schulungen, in denen sie lernte, die Krankheit ihres Mannes zu verstehen und ihn in seiner Erkrankung zu respektieren. Die Beschreibungen ihrer Erfahrungen im Brief und die Bilder ihres Mannes auf der Einladung zur Vernissage weckten bei uns das Interesse, mehr über den Krankheitsverlauf des Mannes zu erfahren, den Frau L. so intensiv begleitet hatte. Dass Frau L insbesondere im fortgeschrittenen Stadium der Demenzerkrankung professionelle Unterstützung in Anspruch genommen hatte, löste bei uns die Frage aus, wie sie die pflegerische Betreuung ihres Mannes und die professionelle Unterstützung erlebt hatte und was sie im Rückblick als wichtig erachtet. Wir fragten Frau L in einem Telefongespräch, ob sie bereit wäre, uns auch persönlich von ihren Erfahrungen zu berichten. Wir vereinbarten mit ihr, dass wir uns erst die Vernissage ansehen und dann ein gemeinsames Gespräch führen würden.

Wir besuchten Frau L. auf der Vernissage und schauten uns ihre Bilder an. Die Ausstellung stellte sich uns als starkes Medium dar, wirkte sie doch so, als setze Frau L. (bewusst oder unbewusst) die Fotografie gegen das Vergessen ein, welches vielleicht das unfassbarste und entscheidendste Symptom dieser Erkrankung ist. Die Prăsentation ihrer Bilder bestatigte unser Gefühl, dass der Krankheitsverlauf ihres Mannes für sie mit Höhen und Tiefen verbunden war. Als sich die meisten Gáste verabschiedet hatten, hatten wir Zeit für ein persönliches Gespräch und ließen Frau L. erzăhlen.

\section{Methodische Aspekte}

Wir fuhrten mit Frau L. ein narratives Interview durch (Lamnek 2005). In der Vorbereitung und während der Durchführung des Interviews berûcksichtigten wir die vier Prinzipien qualitativer Forschung: Offenheit, Kommunikation, Naturalistizităt und Interpretativităt (ebd.). Im Gesprăchsverlauf orientierten wir uns dabei an der Einstiegsfrage: „Wie haben Sie den Krankheitsverlauf Ihres Mannes erlebt?" 


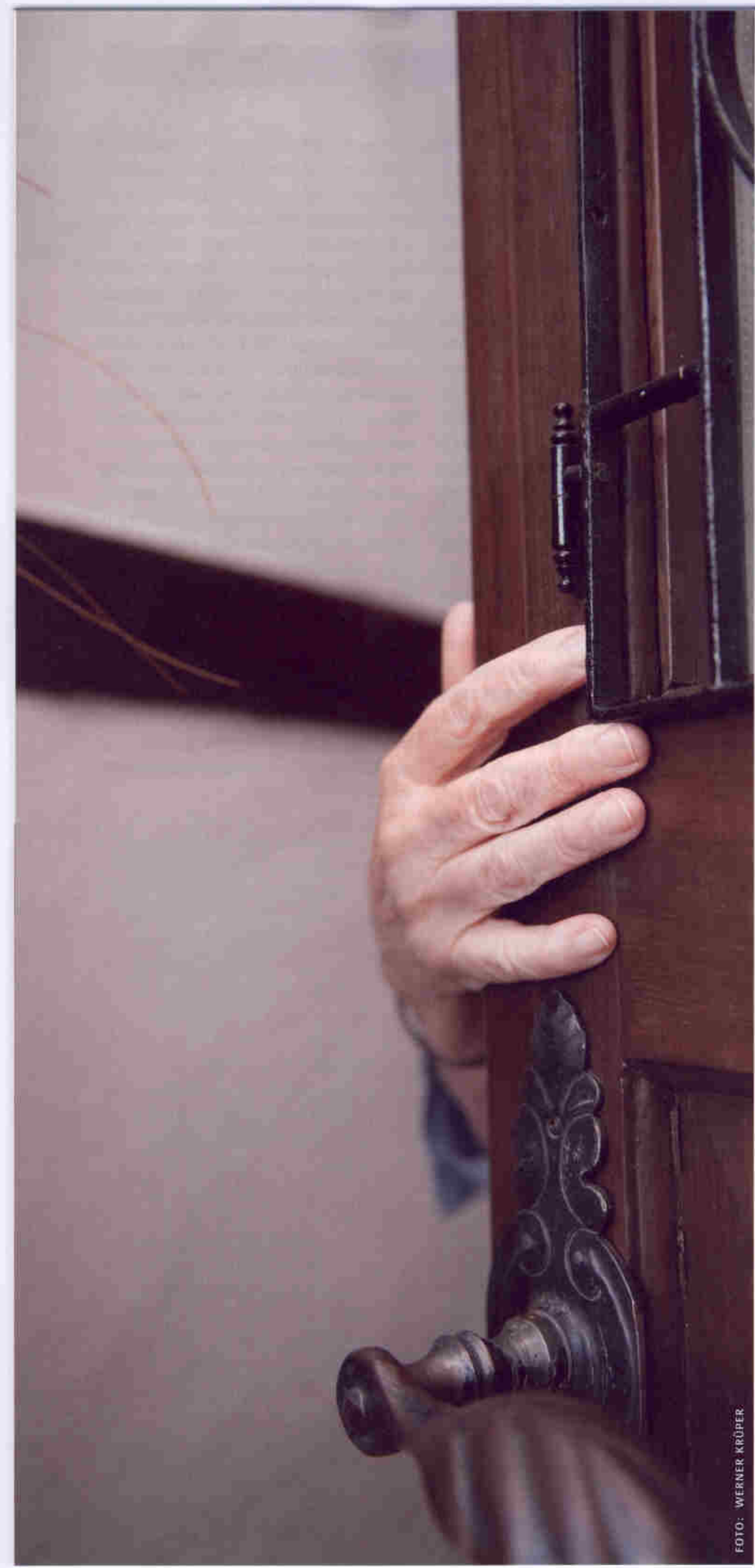

Wir haben das Interview im Einverständnis mit Frau L. digital aufgezeichnet. Wir sicherten Frau L auch nach dem Interview Transparenz zu und tauschten mit ihr Telefonnummern aus, um Unklarheiten oder aufkommende Fragen von Frau L. direkt beantworten zu können.

Wir transkribierten das Interview, fassten nach mehrmaliger Durchsicht bedeutsame Inhalte zusammen und strukturierten einzelne Passagen in Kategorien, die wir gegenseitig auf Stärke prüften, was in Anlehnung an die Inhaltsanalyse nach Mayring (2003) erfolgte. Wir legten Frau L. unseren Bericht zur Durchsicht vor und ließen sie prüfen, ob unser Bericht ihre Erfahrungen wiedergibt und sie sich mit ihren Aussagen identifizieren kann. Es zeigte sich bei den zwei Nachbesprechungen, dass sich Frau L. wohler fühlte, wenn sie von Schwerpunktthemen anstelle von Kategorien sprechen konnte. Sie hatte die Erkrankung ihres Mannes sehr facettenreich erlebt und es war ihr wichtig, die Erinnerungen ihrer Erlebnisse aus dem Krankheitsverlauf annähernd chronologisch darzustellen. In Absprache mit ihr wurde der Anfangsbuchstabe ihres Namens durch einen anderen ersetzt.

\section{Ergebnisse}

Nachdem wir das Interview transkribiert hatten, haben wir es in Abstimmung mit Frau L. auf sprachliche Feinheiten verbessert. Aus der Analyse des Interviews mit Frau L. kristallisierten sich die vier Schwerpunktthemen „Sich einsam und überfordert fühlen”, „Ohne Hilfe ging es nicht", "Lernen, mit der Erkrankung umzugehen" sowie „Kommunikation und Zusammenarbeit" heraus.

\section{"Sich einsam und überfordert fühlen"}

Besonders in den Anfängen der Erkrankung, in der Frau N. ihren Mann zu Hause pflegte, gab es noch kein Netz, keine Vertrauten oder Gleichgesinnten für Frau L, mit denen ein Austausch über die Demenzerkrankung ihres Mannes möglich gewesen wäre. „Da war viel Verzweiflung drin und Wut über die Veränderung durch die Krankheit, ja Hilflosigkeit", so schreibt sie in einem Brief. Ohne in dieser Zeit bereits 
genau zu wissen, worum es sich bei den Symptomen ihres Mannes genau handelte, erfuhr sie etwas wie Stigmatisierung. Frau $L$ fragte sich. wie wohl die anderen das erleben. Auch waren Gefühle von Oberforderung vorhanden, als ihr Mann vieles von dem, was er abgelegt hatte, nicht mehr wiederfand und er sich auch in seiner Persönlichkeit veränderte:

„Er war doch immer so lieb, der hat mich auf den Händen getragen und jetzt fängt er doch nicht plötzlich an, mich extra zu ärgern."

Die Situation, alleine mit ihrem Mann zu sein, löste aber auch Gefühle von Einsamkeit aus, weil sie sich mit der Erkrankung ihres Mannes unverstanden und vor allem in den Anfängen der Erkrankung alleine fuhlte. Mit ihm selbst konnte sie nicht über seine Erkrankung sprechen:

"Ich habe mit meiner Nachbarin gesprochen und sagte, wissen Sie, es tut mir leid, wenn es vielleicht einmal etwas laut ist. Vielleicht denken Sie ja, diese zwei, jetzt gehen sie wieder friedlich Arm in Arm. Aber es ist ja die Krankheit. Ich wollte irgendwie etwas erklären und die hat mir geantwortet: Das könnte ich nicht, wenn mein Mann und ich uns streiten, dann dauert es Tage, bis wir uns wieder vertragen. Die hat gar nicht begriffen, was ich sagen wollte."

Entscheidend bei der Verarbeitung der Situation war das Verständnis für die Erkrankung. Informationen zum Krankheitsbild ermöglichten Frau L., die Krankheit zu begreifen und Möglichkeiten zu nutzen, um damit umzugehen:

"Das ist im Moment wie eine Erlösung, die Diagnose zu erfahren - er hatte also wirklich etwas. Also ich spinne nicht und er spinnt nicht - das habe ich begriffen. Dann habe ich auch Informationen erhatten für Tagesheime und so und ich habe gedacht, ja. das kommt noch lange nicht in Frage - aber das sagen die meisten, mein Mann will doch etwas nicht. Aber das war dann die Rettung. Da hatten wir nachher wirklich die Hilfen und die soziokulturellen Angebote, was uns sehr viel gegeben hatte"

Oberfordert fühlte sich Frau L. in der Anfangszeit der Demenzerkrankung, als sie sah, dass ihr Mann bei seinem ersten Klinikaufenthalt die Medikamente nicht vertrug:
„Man hat ihn beruhigen wollen und er hat paradox auf die Medikamente reagiert. Er ist fast die Wände hochgegangen. Er war vorher so rüstig, und dann konnte er schon nach einem Monat nicht mehr gehen, geschweige denn Treppen steigen. So war es mir nicht möglich, ihn nach zwei Monaten nach Hause zu nehmen."

Dank des leitenden Arztes entlasteten Frau L. freiwillige Helfer, indem sie mit ihrem Mann drei Wochen nach Davos gingen. So konnte Frau L. wieder Kraft schōpfen. Allerdings war die Situation für sie so "krass", dass sie einsehen musste, ihn auch anschließend nicht nach Hause nehmen zu können, weil sie das ūberfordert hä̀te.

\section{„Ohne Hilfe ging es nicht"}

Als ihr Mann mit den freiwilligen Helfern in Davos war, musste sich Frau L. in der für sie „unseligen Zeit” etwas einfallen lassen. Sie wusste, dass sie handeln musste und dass ihr Mann mit dem Eintritt in ein Heim Ende der 1990er-jahre ,festgenagelt" wurde. Für Frau L. war dann nach der Klinik das zweite „schockartige“ Erlebnis, als sie auf dem Bildschirm des Computers das „festgenagelte" Datum des Eintritts sah:

„Ich habe gespürt, er weiß, er muss da rein und kommt nicht nach Hause. Traurig war es - aber ich wusste ja, es geht nicht anders."

Für Frau L. war die Heimunterbringung schwer, vor allem weil sie das Gefühl hatte, ihn „einzuteilen”, ja über ihn zu verfügen:

„Das war schrecklich, aber ich wusste auch, dass es keine andere Lösung gab. Dank dem Konzept des Heimes ,Pendelbett' hatte ich einige Zeit später die Möglichkeit, meinen Mann in regelmäßigen Abständen nach Hause zu holen."

Frau L konnte dank dem „Pendelbett” vor allem in der Anfangszeit der Erkrankung zu einer Kur ins Appenzeller Land fahren:

${ }_{n}$ Er ist ja von dort und hatte zu seiner Freude täglich das ganze Alpstein-Gebirge vor dem Fenster, wo wir auch täglich wanderten, große Spaziergänge machten in der Schneelandschaft, was sowohl ihm als auch mir sehr gefiel. Für das Essen war gesorgt und wir waren in einer guten Pension, in einem Hotel, im höchsten Dorf des Kantons."
Nach schon zwei Monaten nahm Frau L ihren Mann immer häufiger nach Hause, bis er dann nur noch eine Nacht in der Woche im Heim verbrachte:

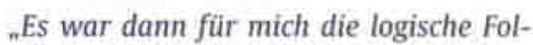
ge, thn ganz herauszunehmen, obwohl es dann doch nicht so gut lief wie erhofft. Als ich ihn da herausnahm, musste ich ein paar Tage später unterschreiben gehen, dass ich das verantworten könne. Ich ging mit den blauen Armen [...] das durfte der Arzt nicht sehen, weil ich sagte, es gehe gut!"

Für Frau L. lief es dann doch nicht so gut, wie sie erhofft hatte, was dazu führte, Herrn L. wieder notfallmäßig unterzubringen:

„Die letzten Erfahrungen waren sehr schmerzlich für mich. Ich habe es in den letzten zwei Monaten vor seinem Tod nicht mehr geschafft, wie ursprünglich gewünscht, ihn bei mir zu behalten. Das ist die Zeit, wo's einem ohnehin beinahe das Herz bricht [...] nochmals diesen geliebten Menschen aus der Wohnung tragen lassen ,zu müssen', an einen anderen Ort bringen lassen zu müssen, quasi Heimeintritt auf der Todesbahre! Ich darf da nicht mehr daran denken, sonst wird mir übel $[. . .]^{*}$

\section{„Lernen, mit der Krankheit umzugehen"}

Die Persönlichkeitsverănderungen ihres Mannes forderten Frau L. heraus, ihr eigenes Handeln gegenüber ihrem Mann zu reflektieren und zu hinterfragen:

„Einmal hat er gesagt, das ist ja das Dramatische - als er sa $\beta$ und ich stand, da sagte er: Ich komme ja gar nicht mehr gegen Dich auf! Wow! Das hat mich getroffen. Ich dachte, das musst Du Dir merken, das darfst Du nie mehr vergessen, Du musst so mit ihm umgehen, dass dieses Gefühl bei ihm nicht vorherrscht."

Frau L erinnert sich an Situationen, in denen sie ihrem Mann gerne mehr Zuwendung geschenkt hätte, dies aber aufgrund der Umstănde nicht konnte:

„Und dann hat er mal etwas gesagt, eine Bemerkung - da habe ich gesagt: Wie bitte? - Ja, Du hast schon gemerkt, dass da oben etwas nicht mehr stimmt, hast mich nicht mehr gerne! - Und da hätte ich einfach hingehen sollen, können, und ihn umarmen und sagen, ja natürlich lieb ich Dich genauso und ich konnte das nicht." 
Auf die Frage, was gute Tage waren, war es vor allem das Verständnis anderer, das sie schätzte, aber auch der Austausch mit ăhnlich Betroffenen:

„Wenn uns andere so genommen haben, wie wir sind, dann habe ich sagen können, es war ein guter Tag - einfach so, wie wir sind. Man merkt schnell, wenn andere das Verständnis nicht haben. Wenn andere sich Zeit genommen haben, mit uns zu reden wenn man Isolation kennt. gewinnt das an Bedeutung."

Profitieren konnte Frau L. von der Vielzahl an soziokulturellen Angeboten, um sich mit der Erkrankung und ihren Auswirkungen auf ihr Familienleben auseinanderzusetzen:

„Geholfen haben uns Tanz- sowie Spaziernachmittage. Feierabendmusik mit dem Singen vieler alter, bekannter Lieder. Referate mit Weiterbildungswert und die anschließenden gemeinsamen Mittagessen, wie eine Familie, erst 14-täglich, später monatlich. Uns wurde praktisches Wissen weitergegeben, z.B. über das Malen mit Demenzerkrankten und über Musik als Therapie sowie das Tier als Therapeut: Katzen, Hunde etc."

Frau L. berichtet von den Schulungen und dem persönlichen Gewinn an Fachwissen und dem Gemeinschaftsgefühl, welches die Situation einfacher machte:

„Die ganzen Ausstellungen mit Vorträgen uber das Gehirn mit Film und grafischen Darstellungen. Ganze Podien über Hirnverletzte, Epilepsie. Es gab Schiffsausfahrten eigens für Alzheimer-Betroffene mit ihren Angehörigen, wobei für jedes Paar, oder Mutter-Tochter oder-Sohn eine Betreuerin zugeteilt wurde, so war es eine echte Entlastung und ein gemeinsames freudiges $\mathrm{Er}$ lebnis. Aber auch die Schulungen, in denen ich lernte, dass ich meinen kranken Partner nicht ändern noch erziehen kann. Ich kann nur mich ändern, anpassen, versuchen. nicht anzustoßen."

Frau L. machte Kurse in Kinästhetik und besuchte Angehörigengruppen:

„Angehörigengruppen sind etwas vom Allerwichtigsten. So vieles bin ich nur dort inne geworden, so vieles, was ich nirgends sonst hätte in Erfahrung bringen können, wurde dort erörtert. Informatives wie auch Probleme, wie man die lōsen kann. Alle und jeder wusste, von was wir sprechen, und haben Ähnliches erlebt, auch Verständnis und oft Hilfen und Ideen, was zu tun wäre."

Für Frau L. war es wichtig, gewisse Gewohnheiten ihres Mannes zu pflegen. So hatte sie bei Unternehmungen seine Mundharmonika dabei:

"Meine Erfahrung war es, dass Wandern kombiniert mit Schiffsfahrten ungemein gut tun. Wir waren enorm oft auf dem Rhein. Erst wandern, dann auf dem Schiff ausruhen. Immer war die Mundharmonika dabei. Er hat auch getrommelt im Wald und immer war er am Pfeifen, das hat dann jeweils seine Stimmung angegeben, so wusste ich auch immer, wo er war."

\section{„Kommunikation}

\section{und Zusammenarbeit"}

Als die Demenzerkrankung von Herrn L. bereits weit fortgeschritten war und Frau L. ihn regelmäßig im Heim besuchte; war es ihr wichtig, dass sich Pflegende für die Biografie ihres Mannes interessierten, um seine Verhaltensweisen richtig einschătzen zu können:

„Ein Pflegender hat zum Beispiel gesagt: Der pfeift jal - Ja, der pfeift, sagte ich, der ist fröhlich. - Ja, der ist ja ganz euphorisch. sagte er. Ich habe gesagt, wissen Sie, das war immer ein fröhlicher Mensch. Der hatte immer frei am Montag, das ist ein glücklicher Mensch gewesen."

Frau L erlebte, dass Pflegende sich während der Versorgung ihres Mannes in einer fremden Sprache unterhielten, die weder sie noch ihr Mann verstand:

„Es ist wichtig, dass die Pflegenden die Sprache des Patienten sprechen. Also habe ich mal zu zweien gesagt, wissen Sie, jetzt liegt mein Mann da und Sie sprechen da so privat miteinander. Mein Mann kann kein Wort verstehen, was Sie sagen. Also ich wollte damit sagen, sprecht doch ein bisschen mit meinem Mann, wenn ihr was macht, und sagt, wir machen Ihnen jetzt gerade etwas Normales, machen jetzt das und wir wechsein das so und so."

Als pflegende Angehörige wusste Frau L. um die Gewohnheiten ihres Mannes, um die Vertrăglichkeit sowie um die Unverträglichkeiten verschiedener Materialien, und es war ihr wichtig, dies auch zu kommunizieren. Gleichzeitig war sie es von zu
Hause gewöhnt, ihren Mann zu unterstützen und ihn zu pflegen, was ihr aber in der Klinik kurz vor dem Tod ihres Mannes verboten wurde:

„Mein Mann lag nass, als ich kam, ja gar verstuhit, also konnte ich ihn nicht aufrichten mit dem Kopfteil, um ihm zu trinken oder ihm das bereitstehende Dessert zu geben, er wäre ja abgerutscht. Also wechselte ich ihm mal die Unterlage, wechselte ihm das Hemd, versuchte auch die Pants zu wechseln, bevor jemand eintrat - was ich ja zu Hause auch täglich $x$-mal gemacht hatte."

Frau L. hatte gehofft, mit den Pflegenden das Material, das für ihren Mann verwendet wurde, abstimmen zu können:

„Das Problem mit der Inkontinenz und mit dem Material war, dass es einfach immer irgendwo durchgeronnen ist, und das habe ich nicht einfach hingenommen und habe die Unterlagen gewechselt und so."

Frau L wollte ihre Erfahrungen, die sie zu Hause mit ihrem Mann gemacht hatte, gerne weitergeben, die Zusammenarbeit mit den Pflegenden war ihr sehr wichtig:

„Ich bin zum Arzt gegangen, der die Praxis hat und das Heim betreut, und habe ihn gebeten, dass er denen den Auftrag gibt, wieder die anderen Inkontinenzmaterialien zu nehmen. Das ist ja grauenhaft, wenn sie am Schluss sehen, was ein Mensch noch durchmachen muss. Und ich habe dem Pfleger gesagt, schauen Sie bitte, ich darf ja nichts mehr machen, schauen Sie bitte, ob es etwas besser geworden ist - so abhängig war ich vom guten Zuspruch."

\section{Überlegungen für die Praxis}

Frau L. hat mit dem Erzählen ihrer Erfahrungen deutlich gemacht, dass es mit dem Fortschreiten der Demenzerkrankung für pflegende Angehörige immer wichtiger wird, geeignete Ansprechpersonen zu finden, die im Verlauf der Erkrankung Unterstützung leisten - sei es durch gezielte Informationen oder aber durch die Übernahme pflegerischer Aufgaben, um Gefühlen wie Einsamkeit und Überforderung entgegenwirken zu können. Die Situation von Frau L. hat gezeigt, dass besonders in der Anfangsphase der Alzheimer-Erkrankung die Einsamkeit pflegender Angehöriger hoch ist und einen Leidensdruck auslöst. weil wichtige Informationen fehlen. Pro- 
fessionell Pflegende sollten Pflegewissen an die Angehörigen weitergeben, am besten individuell und auf die Situation zugeschnitten, um die pflegenden Angehörigen in ihrer jeweiligen Situation abholen zu können. Frau $L$ fühlte sich im Krankheitsverlauf ihres Mannes immer stärker unterstützt, was bestätigt, dass die Angehörigenarbeit in Langzeitinstitutionen bereits seit Längerem ein wichtiges und viel diskutiertes Thema darstellt.

Frau L. erlebte die Persönlichkeitsveranderung ihres Mannes, vor allem aber auch, dass sie mit ihm selbst nicht über die Erkrankung sprechen konnte. Dieser Umstand löste Einsamkeit bei ihr aus und das Gefühl, mit der Erkrankung ihres Mannes alleine zu sein. Professionell Pflegende kōnnen pflegenden Angehörigen im Deuten von Emotionen helfen und ihnen Strategien zum Umgang mit schwierigen Situationen vermitteln.

Wie Frau L. selbst beschrieben hat, halfen ihr die Schulungen und Weiterbildungen im Umgang, aber auch im Verstehen der Demenzerkrankung. Pflegende Angehörige wie Frau L. können beispielsweise auch von Pflegenden dazu angeleitet werden, mit unruhigem oder aggressivem Verhalten Demenzerkrankter umzugehen. Die Resultate der Untersuchung von Perren und König (2005) belegen, dass eine Schulung von Angehörigen im Umgang mit der Demenzerkrankung insbesondere auf der psychosozialen Ebene positive Effekte erzieit. Schulungen tragen demzufolge dazu bei, dass sich das emotionale Wohlbefinden erhöht und die Lebensqualităt erhalten bleibt.

Frau L. fand es besonders wichtig, Pflegenden von der Biografie ihres Mannes zu berichten, ihr war es wichtig, das Pflegende Verhaltensweisen entsprechend deuten und somit einen verstehenden Zugang erlangen. wie sich das bei Frau L. beispielsweise am Pfeifen ihres Mannes gezeigt hatte. Die Bedeutsamkeit von Biografiearbeit in der täglichen Praxis wird durch das Psychobiografische Pflegemodell nach Böhm (1999) aufgezeigt. Böhm (2009) geht von der Auffassung aus, dass eine an den Bedürfnissen orientierte Pflege nur über das Verstehen der Biografie möglich ist und dass erst das "Eintauchen" in die Le- bensgeschichte es Pflegenden ermöglicht, eine therapeutische, verstehende Haltung einzunehmen, die sich reaktivierend und symptomlindernd auf die Situation geriatrischer Patienten auswirken kann.

Anhand der Erfahrungen von Frau $L$. wird deutlich, dass pflegende Angehōrige, die vor allem bereits zu Hause aktiv waren und die Pflege und Betreuung von Demenzerkrankten übernommen haben, häufig den Wunsch äußern, im Verlauf der Alzheimer-Erkrankung weiterhin aktiv zu sein, was durch die Ausführungen von Daneke (2000) bestätigt wird. Sie möchten als Partner anerkannt und in den pflegerischen Alltag einbezogen werden, was auch Frau $\mathrm{L}$ mit ihrem Wunsch nach $\mathrm{Zu}$ sammenarbeit zeigte, um die Pflege ihres Mannes zu optimieren.

Pflegende Angehörige überfordern sich hăufig. was auch die Erfahrungen von Frau L. gezeigt haben. Dass sie sich und ihrem Mann das Sterben zu Hause nicht ermöglichen konnte, beschäftigt sie auch heute noch. Es ist wichtig, Angehörigen das Gefühl zu geben, dass es nicht ihr persönliches Versagen ist, wenn sie die Pflege und Betreuung zu Hause nicht mehr alleine bewältigen können. Viele Angehörige haben den Wunsch, Demenzerkrankte zu Hause zu betreuen und pflegerisch zu versorgen, andererseits kann die pflegerische Versorgung Demenzerkrankter sehr komplex und anspruchsvoll sein und ist besonders im fortgeschrittenen Stadium der Erkrankung in den wenigsten Fällen alleine zu bewältigen. Denn oftmals bedeutet die Pflege von Demenzerkrankten für pflegende Angehörige Zeitdruck, schlechten Schlaf, aber auch eine große psychische Belastung. Pflegende Angehörige werden auch als die „unsichtbaren zweiten Patienten" beschrieben. Die Betreuung im familiâren Umkreis beeinflusst zwar positiv die Lebensqualität des betroffenen Familienmitglieds, stellt für die Erbringer dieser Pflegeleistung aber eine große Belastung dar. Brodaty und Donkin (2009) kommen in einer Obersichtsarbeit zu dem Schluss, dass durch die Form der familiăren Pflege das Risiko einer psychischen Erkrankung zunimmt.

Frau L. hatte sich im Krankheitsverlauf ihres Mannes gewünscht, sich bei pflege-

\section{Das Rätsel}

Ist es nicht seltsam ...

dass, was wir lange erlebten

als Last -

die Pflege eines kranken Menschen die Pflege, die lange dauerte, lang und länger - jetzt vorbei ist?

Es war ermüdend, einengend. entmutigend und manchmal erniedrigend.

Jetzt - auf seltsame Weise -

fühle ich keine Erleichterung.

\section{Vielleicht unabsichtlich.}

gab er meinem Leben einen Sinn einen Sinn, den ich weder verstand

noch zu schätzen wusste.

Jetzt brauche ich einen neuen Sinn

(und dafür bin ich alt genug).

Der Vertrag wurde eingehalten

so gut ich es konnte -

Aber mein Leben geht weiter.

Hilf mir, einen neuen Sinn zu finden eine neue Lösung -

und lass es ein Leben werden,

das nicht nur

gerade ein Weiterleben ist.

(Maude S. Newton)

bezogenen Entscheidungen einbringen zu können. Sie wusste um die Gewohnheiten ihres Mannes und kannte die Bedürfnisse aus der eigenen pflegerischen Erfahrung. die sie über viele Jahre gesammelt hatte. Auch die Ausführungen von Ugolini (2006) machen deutlich, dass pflegende Angehörige den Wunsch haben, sich einzubringen und in gewisser Hinsicht Einfluss nehmen zu können. Weil diese Bedürfnisse im All- 
tag häufig nicht berücksichtigt werden können, schwanken die pflegenden Angehörigen häufig zwischen Resignation und Protest. Es ist daher sehr wichtig, regelmäßige Gesprăche mit Angehörigen zu führen und sie in den pflegerischen Alltag zu integrieren, damit sie die Erkrankung und die damit verbundene Belastung bewăltigen können. Neben dem persönlichen Gespräch gibt es die Möglichkeit, die Qualität der Zufriedenheit von Angehörigen mithilfe des Assessment-Instrumentes OLA (Optimierung der Lebensqualität im Alter) zu erfassen und die Pflegequalität in Institutionen zu verbessern. Es handelt sich um ein Instrument, das valide und reliabel darüber Auskunft gibt, inwieweit die Zufriedenheit von Angehörigen von Altersund Pflegeheimbewohnern vorhanden ist (Huber 2008).

\section{Ausblick}

Als wir Frau L. fragten, wie der Krankheitsverlauf noch besser hätte gestaltet werden können, überlegte sie kurz und sagt dann, dass ihr Mann am Tisch immer etwas getan habe und dass es wichtig sei, über Gewohnheiten und Rituale Bescheid zu wissen, um ihnen im Alltag gerechtwerden zu können, um damit einen Bezug zu gewohnten Lebensweisen herzustellen. Beispielsweise hätte es Herr L, gerne gehabt, in Zeitschriften herumzublättern oder Äpfel zu schälen. Wir fragten Frau L. was ihr abschließend zu unserem Bericht und ihrer Geschichte wichtig sei, den Lesern mitzuteilen:

„Ich spürte, dass da bei ihm noch viel mehr vorhanden war, als allgemein angenommen wurde, da war ich (und bin es jetzt immer noch) zutiefst dankbar, dass ich das erleben durfte. So wie wir im Tod vereint sein dürfen, ich bei ihm sein durfte, ihm einen letzten Liebesdienst tun durfte mit dem Einreiben des Rückens und dem Massieren des Knies, und er so eigentlich unter meinen Händen „davongehen durfte". Das begleitet mich das ganze weitere Leben lang, dafür bin ich ihm unendlich dankbar. Das war bzw, ist das größtmögliche Abschiedsgeschenk, das er mir überhaupt machen konnte! "

\section{LITERATUR}

1 Brodaty H, Donkin M. Family caregivers of people with dementia. Dialogues Clin Neurosci, 2009; 11/2: 217-228

2 Böhm E. Psychobiografisches Pflegemodell nach Bōhm. Band 1: Grundlagen. Wilhelm Maudrich. Wien/München/Bern. 1999 3 Böhm E. Verwirrt nicht die Verwirrten. Neue Ansäzze geriatrischer Krankenpflege. 14. Aufl. Psychiatrie Verlag. 2009 4. Bundesamt für Statistik: Medienmitteilung 14. Gesundheit: Todesursachen des Jahres 2007, 2009. [http://www.bfs.admin. $\mathrm{ch} / \mathrm{bfs} /$ portal/de/index/themen/14/22/press. Document.118950.pdf, Zugriff 17.01.2010] 5 Corbin JM. Strauss AL. Weiterleben lernen. Chronisch Kranke in der Familie. In: Welter-Enderlin R, Hildebrandt B (Hrsg.). Familienwelten (Band III). Pieper, München/Zürich. 1993

6 Daneke S. Angehörigenarbeit. Urban \& Fischer, München. 2000

7 Dibelius 0 , Uzarewicz C. Pflege von Menschen höherer Lebensalter. Grundriss Gerontologie, Band 18. Kohlhammer, Stuttgart. 2006

8 Höpflinger $\mathrm{F}$. Angehörigenpflege - Bedeutung und Entwicklung. Managed Care, 2004: 3

9 Huber E. OLA: Optimierung der Lebensqualität im Alter: Entwicklung eines Fragebogens zur Zufriedenheit der Angehörigen von Bewohnerinnen in Altersinstitutionen. Pflege, 2008; 21/5: 319-326.

10 ICD-10-Code F00: Demenz bei Alzheimer-Krankheit. [http://www.dimdi.de/static/de/klassi/diagnosen/icd10/htmlamtl] fr-icd.htm?gfo0.htm, Zugriff 26.01.2010]

11 Kofahl C, Lüdecke D, Döhner H. Der Einfluss von Betreuungsbedarf und psychosozialen Determinanten auf Belastung und Wohlbefinden von pflegenden Angehörigen alter Menschen. Ergebnisse aus der deutschen Teilstichprobe des Projekts EUROFAMCARE, Pflege \& Gesellschaft, 2009; 14/3 12 Lamnek S. Qualitative Sozialforschung. Lehrbuch. 4., vollst, überarb. Aufl. Psychologie Verlags Union, München/Weinheim. 2005

13 Mayring P. Qualitative Inhaltsanalyse. Grundlagen und Techniken. 8. Aufl. UTB, Stuttgart. 2003
14 Oppikofer S. Temporäre Entlastung für pflegende Angehörige von Menschen mit Demenz. Zentrum für Gerontologie, Zurich. 2009

15 Perrar KM, Sirsch E, Kurschke A. Gerontopsychiatrie für Pflegeberufe. Thieme, Stuttgart. 2009

16 Perren S, König M. Welche Wirkung hat Angehōrigenschulung auf die Betroffenen? In: Wettstein A, König M. Schmid R. Perren S. Belastung und Wohlbefinden bei Angehörigen von Menschen mit Demenz. Eine Interventionsstudie, Rüegger, Zürich. 2005

17 Schulz M, Dorgerloh S, Ratzka S, Gray R, Behrens J. Compliance und Adherence. Pflegende unterstützen Patientenverhalten. PADUA, 2007: 3

18. Schweizerische Alzheimervereinigung: 102000 Menschen mit Demenz in der Schweiz, 2009, |http://www.alz. ch/d/pdf/schweiz_2008_d.pdf. Zugriff 17.01.2010]

19. Ugolini B. Brülke zum „richtigen Leben" - Angehörigenarbeit in der institutionellen Altersarbeit. Psychoscope, 2006; $4: 5-8$

Mag. Daniela Hândler-Schuster Diplom-Berufspầdagogin (FH)

Kontaktadresse:

ZAG Zentrum für Ausbilidung im Gesundheitswesen Kanton Zürich

Turbinenstrasse 5 $\mathrm{CH}-8400$ Winterthur daniela.haen tenwzagran

Dr. rer. medic. Michael Schulz MPH

Kontaktadresse:

Kilinik für Psychiatrie und Psychotherapie in Bethel

Evangelisches Krankenhaus Bielefeld

Remter Weg 69-71.

33617 Blelefeld

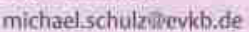

Bibliografie

DOI 10.1055/5-0030-1251964

Psych Pflege 2010: 16: 75-82

- Georg Thieme Veriag KG

Stuttgart - New York - ISSN 0949-1619 